\title{
RECUPERAR LA RETÓRICA: T.S. KUHN Y LAS CIENCIAS SOCIALES
}

\section{J. Francisco ÁLVAREZ}

"En la práctica científica, tal como se comprueba en la literatura académica, el científico más bien parece que está batallando con los datos intentando forzarlos para que se adapten a una teoría de la que no tiene ninguna duda" ( T. S. Kuhn, The Essential Tension, 1977, pág. 193)

La amplia difusión que tuvo la obra de Kuhn entre los científicos sociales durante los años que median entre 1965 y 1980 demanda un análisis que vaya más allá de la descripción sociológica. Para tratar de explicar un fenómeno tan excepcional podría ser útil echar mano de ciertos procedimientos metarreflexivos, por ejemplo, intentar aplicar el pensamiento de Kuhn para explicar la influencia de Kuhn en la ciencia social. Si siguiésemos esa orientación aparecerían diversos problemas de legitimidad, los internos a la comunidad de los científicos sociales y a las diversas fases de incorporación de las propuestas kuhnianas, y sería conveniente realizar el seguimiento del desarrollo posterior.

Aparecen así al menos dos líneas de trabajo a explorar. La primera consiste en analizar la presencia de Kuhn en los libros de texto de psicólogos, antropólogos, economistas, etc., particularmente entre quienes no hacen explícitas reflexiones metodológicas. Es fácil comprobar que Kuhn ocupa en el ámbito de las ciencias sociales un lugar que se corresponde con el que se suele asignar al método inductivo en los primeros capítulos de los libros de texto de física; aparece la referencia a Kuhn para mostrar que trabajan en un campo cuya cientificidad viene garantizada por la

Éndoxa: Series Filosóficas, $n^{\circ}$ 9, 1997, UNED, Madrid:

J. Francisco Álvarez: Recuperar la retórica: T. S. Kuhn y las ciencias sociales. pp. 167-186. 
conformación de un paradigma, en el seno del cual se propone desarrollar la actividad y en el que se aconseja que se formen los jóvenes investigadores.

La segunda vía, cuyo análisis puede tener algún interes, consiste en comprobar cómo desaparece el papel del Kuhn historiador de la ciencia y, por el contrario, cómo se utilizan sus propuestas epistémicas para clausurar la utilización de la historia de las correspondientes disciplinas y para borrar las huellas de los padres fundadores. Reflexiones como las que se encuentran en el trabajo "La centralidad de los clásicos" de J. Alexander (A. Giddens y otros: Social Theory Today, 1987) son un buen ejemplo de esa utilización multívoca de Kuhn.

Como se ha dicho por parte de destacados filósofos de la ciencia social (v.g., Martin Hollis, The philosophy of social science, Cambridge, 1994) la historia del "libro" de Kuhn ejemplifica su propia tesis: Pensar en la ciencia y en el conocimiento en general de una nueva forma, puesto que los inevitables paradigmas que regulan la ciencia normal no están sujetos a una refutación directa. Nos muestra una forma atenta de percibir la dinámica en las ciencias en oposición a las versiones estáticas que dominaban la filosofía de la ciencia.

La sorpresa y los efectos del inesperado derrumbe del sistema del socialismo real quizá pudiera servirnos para establecer cierta analogía viva que permita captar el ascenso y declive de determinadas teorias científicas, su supuesto realismo y su pretendida adecuación a "mecanismos objetivos". La analogía misma no está alejada de la propuesta de Kuhn y explicaria algo del enorme predicamento que sus teorías tuvieron sobre los científicos sociales de inspiración marxista.

La obra de Kuhn, además de sus merecimientos propios, refleja sin duda la importancia de decir algo adecuado en el momento preciso. No se trata ahora de localizar toda la complejidad de la propuesta de Kuhn, ni ponernos a matizar las "reflexiones posteriores", los "second thoughts" $u$ otros comentarios y ensayos para diversas enciclopedias. Tampoco para nuestros objetivos vale 
la pena analizar la obra de algunos seguidores muy peculiares como los de la linea Sneed-Balzer-Moulines, pertenecientes a la llamada escuela estructuralista en teoria de la ciencia. La obra de Kuhn en relación con la ciencia social caía en un terreno bien abonado, para el éxito de sus propuestas, tanto en lo social como en lo científico. Si leemos ahora algunos manuales de los años sesenta sobre cibernética o sobre la revolución de los computadores, percibimos (a toro pasado) que la necesidad de romper amarras, de pensar siguiendo otras pautas, de reestructurar los campos perceptivos, de reconceptualizar nuestra experiencia se presentaba como nuevos ámbitos ante la experiencia de los científicos, incluso entre los científicos sociales aunquemostrando éstos menor sensibilidad que los científicos naturales ante los cambios sociales; pero incluso entre los especialistas en ciencia social se producian movimientos y cambios aunque en su gran mayoría siguieran rígidamente encorsetados en torno a la obra de Parsons o en lecturas excesivamente empiristas de Max Weber. De otra parte, en la vida social, en las experiencias políticas, en los acontecimientos cotidianos, se iba produciendo lo absolutamente inesperado, la más rotunda falsación de la capacidad predictiva de las grandes teorías sociales disponibles. Hoy en día ya la única certidumbre es la incertidumbre.

Algunas situaciones avalan nuestra hipótesis del uso oportunista que se hizo de la obra de Kuhn. Ciertos derrumbes posteriormente ocurridos en ciencia social, ejemplos pueden ser el "paradigma marxiano" y los "paradigmas radicales en economía" o el "paradigma conductista en psicologia", que estaban en plena expansión en aquellos años hicieron uso indiscriminado de Kuhn para legitimar productos que difícilmente podían ya entonces sostenerse con los criterios de calidad científica normal (ante resultados de la economía académica más rigurosa o ante las propuestas iniciales de la psicología cognitiva). Analizar todo ese amplio panorama desbordaría los limites de este comentario. Quisiera referirme solamente a otra influencia kuhniana, que me 
parece más positiva y que conecta a la obra de Kuhn con aspectos actuales de la reflexión metodológica en ciencias sociales.

Me refiero al impacto que la obra de Kuhn ha tenido sobre quienes defienden el "giro retórico en ciencias sociales". Muchos defensores de ese giro hacen explícitos sus vínculos con la obra de Kuhn y señalan cómo la obra de Kuhn les sirvió para romper amarras con otras concepciones en filosofía de la ciencia, en particular con las propuestas popperianas. Este es el caso de Donald N. McCloskey en Knowledge and persuasion in economics, Cambridge, CUP, 1994. Ahora bien, aparte de declaraciones explícitas me parece que se da una fuerte conexión interna entre la obra de Kuhn y el giro retórico; una conexión que, para hacerse explícita, supone realizar cierto camino por el análisis de la argumentación y por la teoría de la elección social. Aquí indicaré parte de ese camino sin mostrar punto por punto otras conexiones obvias con la obra de Kuhn.

Es un lugar común, casi una vulgaridad, recordar que los psicólogos desde mucho antes de llegar a su fase conductista se dispusieron a investirse con batas blancas para, con ello, tratar de representar el papel que los físicos habían jugado en las ciencias naturales y defender así, incluso simbólicamente, su experimentalidad, su cientificidad, su acercamiento a las ciencias naturales y su alejamiento de las ciencias humanas.

También se trata de algo común decir que los sociólogos, particularmente los que utilizan las diversas técnicas sociométricas, han pretendido transmitir la supuesta precisión de las matemáticas a los resultados de sus encuestas, a sus investigaciones empiricas, salvo algún que otro contratiempo como el sufrido por el resultado de las últimas elecciones en España o el inadecuado análisis de los comportamientos sexuales en la sociedad estadounidense. Por el contrario, no parece tan común discutirle a la ciencia económica el papel de jefe supremo de las ciencias humanas y sociales, haciendo explícita su retórica construcción argumental. Quizá lo que mejor pueda contribuir a destronar a la ciencia económica de sus 
excesivas pretensiones sean las declaraciones de diversos "expertos" seleccionados en diversos países como asesores gubernamentales para dinamitar el "estado del bienestar". ¿Cómo es posible que la ciencia supuestamente más cercana a la física esté tomando ese papel de combate político central? Para entenderlo me parece importante analizar las construcciones de la economía desde un balance de los recursos retóricos y abordarla con algunas de las técnicas de la crítica literaria. En su trabajo "Entre canibalismos y magnicidios. Reflexiones en torno al concepto de ironía literaria", Ana Rosa Domenella, del Área de Literatura Hispanoamericana de la UAM de México, nos ofrece, quizá de manera no intencional, parte del material preciso para proceder a un cierto tipo de crítica de la economia que señala a la construcción retórica de esta ciencia social.

El recurso literario a la ironía por parte de la ciencia económica tiene nobles antecedentes en el bien conocido tratadillo de Jonathan Swift y su científica "modesta proposición" para resolver el hambre en Irlanda. Aparece también como central en un interesante texto del escritor Julio Torri (1889-1970), "La cocinera", un cuento breve escrito en 1940 que nos presenta una estupenda comida en la que todos los comensales estan satisfechos del nivel gastronómico de las viandas, en la conversación se introduce un personaje "mi vecino de la derecha, profesor de Economía Política, [quien] disertaba con erudición amena acerca de si el enfriamiento progresivo del planeta influye en el abaratamiento de los caloríferos eléctricos y en el consumo mundial de la carne de oso blanco", haciendo explícita la conexión entre ese tipo de ciencia y lo que está ocurriendo en la comida; mientras, una niña, desde luego muy mal educada, irrumpe con impertinencias y con su descubrimiento de "un precioso meñique de niño en su tamal". Además Torri introduce un elemento externo que servirá para corroborar, para añadir evidencia externa probatoria: "Quedó explicada la frecuente desaparición de criaturas en el lugar". En la corrección teórica del estado de bienestar, en la supuesta cientificidad de las propuestas de los expertos, me parece que lo más útil sería mostrar científica- 
mente la utilidad de la eutanasia activa, acabar, por ejemplo, con todos los mayores de 65 años, lo que además produciría una importante expansión del empleo, pues no son pocas las actividades productivas que se generarian al calor de esa modesta proposición.

El último giro quizá sea un camino que no parece científico, no resulta serio, no es lógico, seria simplemente retórico y cargaríamos de nuevo negativamente esa palabra. Así que seamos un poco más serios y abordemos con seriedad el problema. Aunque antes de seguir adelante me gustaría recordar la expresión de Jankelevitch: "ironizar es elegir la justicia".

Hagamos explícito lo que se ha llamado el "giro retórico", estudiemos sus diversas dimensiones y sus correspondientes consecuencias para la actividad científica o, cuando menos, sus consecuencias a la hora de comprender la actividad científica. En el ámbito de las ciencias sociales quizá valga la pena señalar que lo que se conoció como el giro lingüístico es una primera fase de esta otra vuelta de tuerca. Hablar del giro retórico en ciencias sociales es en sí misma una propuesta que tiene mucho de retórica, porque se trata de insinuar y de persuadir con la opinión de que la misma constitución de determinados estudios en disciplinas académicas es el resultado de discursos persuasivos dirigidos a audiencias específicas. Esos discursos pueden haberse dirigido a instancias políticas, a grupos profesionales ya constituidos o quizá hayan reclamado la aquiescencia de la "opinión pública". Como señalan R.H. Roberts y J.M. Good en The Recovery of Rhetoric. Persuasive discourse and disciplinarity in the human sciences, Charlottesville, Virginia, 1993: "esas disciplinas fueron construidas, legitimadas $e$ institucionalizadas mediante ese tipo de discurso persuasivo". Además, muchas de esas disciplinas se mantienen vigentes por procedimientos retóricos, como puede observarse si atendemos a sus discusiones epistemológicas internas o a los debates sobre las distinciones entre áreas de conocimiento. Su autonomía metodológica y conceptual se quiebra porque en todas esas discusiones los discursos son retóricos. La constitución de una 
disciplina no es algo necesario e intemporal, por el contrario cierta unificación de actividades refleja más bien la capacidad de construir discursos capaces de convencer a quienes pueden apoyar esa constitución. Pero, desde mi posición, eso sería simple registro de un uso de la retórica, y de un uso que arrastra cierta valoración de la misma retórica. Sin embargo, los usos retóricos en las ciencias sociales son mucho más amplios; vemos a la retórica cumpliendo su papel en la constitución de las disciplinas pero también sirviendo para formular conocimiento en formas alternativas. Como ha señalado Herbert W. Simons, en un trabajo sobre "la retórica de los proyectos de investigación científica" recogido en el ya citado libro de Roberts y Goods: "Una auténtica virtud del 'giro retórico que se presenta en una gran variedad de disciplinas ha consistido en mostrar las ventajas de combinar la sensibilidad retórica con la competencia académica e investigadora".

Quienes critican este tipo de reflexión repiten palabras y argumentos de J. Locke, aunque con menor contundencia y potencia argumental, perdónese pues la siguiente larga cita: " $\mathrm{Si}$ queremos hablar de las cosas como son, debemos admitir que todo el arte de la retórica, exceptuando el orden y la claridad, todas las aplicaciones artificiosas y figuradas de las palabras que ha inventado la elocuencia, no sirven sino para insinuar ideas equivocadas, mover las pasiones y para seducir el juicio, de manera que no es sino superchería; y, por tanto, por muy laudables o adecuados que puedan ser la oratoria en las arengas y los discursos populares, es cierto que en todos los discursos que pretendan informar o instruir debe ser totalmente evitada; y cuando concierne a la verdad o al conocimiento, no puede sino tenerse por gran falta, ya del lenguaje, ya de la persona que hace uso de ella. Cuáles y cuán varias sean, es superfluo señalarlo aquí; los libros de retórica, abundantes en el mundo, pueden instruir a los que deseen informarse. Solamente no puedo sino observar lo poco que se preocupan de la conservación y el aprovechamiento de la verdad y el conocimiento, ya que las artes de la falacia son las elegidas y preferidas. Es evidente en qué gran medida los 
hombres aman el engaño y el ser engañados, puesto que la retórica, ese poderoso instrumento el error y la falacia, tiene sus profesores establecidos, es públicamente enseñada y ha sido siempre tenida en gran reputación; y no dudo que se tenga por gran atrevimiento, sino por brutalidad, el que yo haya dicho todo lo anterior en su contra. La elocuencia, como el sexo bello, tiene encantos demasiado atractivos para que se permita hablar en su contra. $Y$ resulta inútil intentar buscar los defectos de aquellas artes de engaño cuando los hombres encuentran placer en ser engañados" (J. Locke: Ensayo sobre el entendimiento humano, 3.x.34s).

Locke refleja con bastante precisión la posición general que ha tenido la modernidad sobre la retórica; como ha dicho Richard Lanham "aún estamos aturdidos por aquella gran simplificación que hizo de la retórica una palabra marcada negativamente, pero estamos comenzando a superar y salir de esa fase".

La retórica a fines del siglo veinte se ha tenido que reiventar con cierta ignorancia de su pasado. Los matemáticos que reflexionan sobre la noción de prueba en topología, el economista que se da cuenta de que el Banco Central es un hablante con intenciones o el científico político que se preocupa por las ecuaciones de regresión para ver si la política puede verse reducida al análisis de las encuestas de opinión pública, todos ellos están practicando la retórica. Cuando reflexionan sobre sus reflexiones están prácticando la "retórica de la investigación". Todo esto parece mucho más claro tras una lectura atenta de los artículos de Kuhn que aparecen en la Tensión esencial, en particular el titulado "La función de la medición en la física moderna".

A Kennet Arrow, según recogen diversos autores (Blaug, McCloskey) le preguntaban en 1987 sobre los criterios que utilizaba para evaluar teorías en competencia en economía, contestaba:

"Su capacidad de persuasión. ¿Corresponden a nuestra comprensión del mundo económico? Me parece tonto decir que nos 
apoyamos totalmente en una fuerte evidencia empírica. Una parte muy importante de la evidencia es precisamente nuestra percepción del mundo económico. Si propones un nuevo concepto, la cuestión es si con ello consigues iluminar tus propias percepciones. Si con él sientes que comprendes lo que pasa en la vida cotidiana. Desde luego que es importante si se ajustan a otros test y a los resultados empíricos".

Es curioso señalar que esta frase aparece en el libro de Mark Blaug para decir que Arrow es un lakatosiano. Sin embargo lo que dice Arrow no es sino que él, como todos nosotros, es un retórico. Busca la persuasión, utilizando cierto sentido del mundo social y mediante los mejores test econométricos.

Platón asociaba la retórica con las instituciones democráticas, las asambleas y los tribunales de justicia. En cierta forma malintencionada podemos decir que el ataque contra la retórica tiene cierto contenido antidemocrático. La excelencia del saber parece ir en contra de las decisiones de la asamblea, no es extraño que incluso el sesgo de género aparezca aquí. Las elecciones y los estilos inherentes al discurso científico son argumentos dependientes del contexto y este rasgo debe tenerse en cuenta para poder analizar, comparar y contrastar los diversos tipos de argumentación.

Para mostrar el papel positivo, activo, de la retórica, incluso cuando se pretende que es simple enmascaramiento quisiera comentar un curioso trabajo de finales del siglo XVI. En el año 1596 Felipe II firmó en El Escorial, Madrid, la orden de autorización para la publicación de un libro de Doña Oliva de Sabuco, titulado Nueva Filosofía, Medicina y vera filosofía. Doña Oliva había nacido el 2-12-1562, veinticuatro años antes, en Alcaraz, provincia de Albacete. El texto resulta interesante por diversas razones, no sería la menor decir que lo podrían tener para sí los pedagogos como una buena presentación retórica de su papel profesional y, además, es significativo por los avatares que ha seguido incluso el reconocimiento de su propia autoría, ya que se ha discutido mucho sobre si fue escrito por doña Oliva o por su padre, o por su 
hermano, o por el boticario de Alcaraz, dando como razón principal la evidente y persuasiva de que una mujer con veinticuatro años no puede tener tanto conocimiento como el que aparece en ese texto, $u$ otra razón no menos peregrina como es que lo firmaba ella para evitar la persecusión del Santo Oficio, que al parecer perseguía en aquellos momentos con menor saña a las mujeres que a los hombres. Lo que interesa destacar del libro de doña Oliva es el aspecto positivo de la retórica a que se refiere en el capítulo sexto titulado "De la ira y su remedio, la insinuación retórica". Escrito en forma de diálogo, pregunta uno de los interlocutores : "¿Qué cosa es la insinuación retórica?", a lo que se da la siguiente respuesta: "Es una razón, que quita el enojo como con la mano, y digna de ser sabida en especial cuando el enojo es ira impetuosa por haber reñido con otro y se desea venganza. Dícese insinuación porque el que pone esta medicina se hace de la voluntad, seno y bando del agraviado que quiere curar y dice: "¿Que ese agravio os hizo? ¿esas palabras os dijo?, de la paciencia que teneis me espanto, yo no la pudiera sufrir". Cuando ya esta metido en el seno del agraviado, y ya le da crédito, entonces pone dilaciones al negocio, como, señor no hagais cosas sin mí, mañana, o de aquí a tal hora iremos y tomaremos venganza (...) yo he visto que esta insinuación retórica obra maravillosamente y quita el enojo, y no pasa el daño adelante" (págs. 93-94).

En cierta forma la constitución disciplinar de las ciencias sociales tiene mucho que ver con esa medicina de la insinuación retórica. No se trata del papel de la Retórica a la hora de la simple presentación del conocimiento, sino que los elementos retóricos son constitutivos e incluso son un buen método de curación. Desde luego un método de curación que podría decirse que adelanta a lo que hoy se conoce como diván psicoanalítico, cuando se refiere a la acción directa sobre el supuesto enfermo, pero también sobre la manera de presentación de los resultados, a los informes científicos que aparecen en los libros de psicología -ya sea conductista ya sea cognitiva. El elemento retórico está presente en múltiples aspectos 
de la psicología social, tanto en el diseño experimental cuanto en la presentación de los resultados de la investigación.

Los usos de la estadística tienen más que ver con estos elementos retóricos que con resultados empíricos. Algo que saben bien muchos investigadores, pero que no le resta eficacia y que, en mi opinión, podría beneficiarse de un reconocimiento explícito del componente retórico.

Una manera de insinuar la eficacia del reconocimiento del giro retórico es recordar que por todos lados aparece hoy lo que se conoce como la razón comunicativa, la razón dialógica, o también recordar el enorme papel que jugó la noción kuhniana de paradigma en la presentación de los manuales de ciencia social durante los años setenta.

Con frecuencia se considera que la argumentación resulta opuesta a la demostración. Tenemos asi, por un lado, la demostración sin relación alguna con la retórica y, por otra parte, aparecería la manipulación psicológica sin duda relacionada con la retórica pero nada o muy poco con la demostración. Pero si entendemos la argumentación como un discurso racional que intenta convencer, aunque sea sin el rigor formal de la demostración, entonces aparece el problema de su relación con la retórica.

Algunos temas de la investigación más reciente en la teoría de la decisión, especialmente el estudio de los límites de esa teoría y, sobre todo, el análisis de la toma de decisiones en situaciones de conflicto no resuelto y en situaciones de incertidumbre, no son un simple apartado técnico de esa teoría sino que tienen gran relevancia en diversos terrenos filosóficos; particularmente, tienen importancia para temas referidos a la teoría moral y a la teoría de la investigación científica, tanto a la ética cuanto a la metodología. Me voy a referir exclusivamente a los problemas epistémicos que se pueden derivar de esa reflexión; para ello será conveniente realizar cierto excursus sobre la relación entre la inducción y la argumentación. 
Por ejemplo, Jon Elster ha insistido mucho en que la teoría de la elección social no es capaz de señalarnos un único camino, que con frecuencia no existe el sendero óptimo de elección $y$, en muchos casos, no es único. La importancia de este problema no reside solamente en que si fuese única la opción se anularía la diferencia entre el conjunto de oportunidad y el conjunto de soluciones admisibles, lo que es tanto como decir que no habria elección. Incluso en una situación de determinismo débil deberíamos mantener esa diferencia. Si pretendemos utilizar la teoria como elemento normativo y, a partir de ella, pretendemos explicar causalmente la elección social, estaríamos de hecho ante una contradicción. Se trata de ver que desde el momento en que adoptamos la teoria de la elección estamos restringiendo el ámbito de la explicación causal. Como ha señalado Isaac Levi no podemos quedarnos con los aspectos mejores de las dos opciones. Aquí resulta conveniente reflexionar sobre lo que me gusta llamar el juego de la argumentación inductiva.

El problema central de la inducción es, para nosotros, cómo al añadir nueva información a nuestro cuerpo de conocimiento nos comprometemos a evaluar nuestras opciones cognitivas (nuestras propuestas hipotéticas) con respecto a dos desiderata (seguimos aquí a Isaac Levi) : a) en qué medida esas opciones fomentan la adquisición de nueva información, b) hasta que punto evitan el error. En la inducción se incurre en el riesgo de error; en el esfuerzo por expandir el conocimiento el investigador debe preocuparse por obtener nueva información libre de error, es decir tendrá que evaluar sus opciones cognitivas de manera tal que representen un acuerdo entre el desideratum de evitar el error y el de obtener nueva información. Se trata de un principio general que impone ligaduras o constricciones sobre la manera que las opciones deben ser evaluadas en un cierto tipo de situación.

Destaquemos que es precisamente en el ámbito de la inducción donde aparece con frecuencia la relevancia de los valores éticos, las opciones políticas y los intereses personales de los científicos a la hora de desarrollar su ciencia. Es por esta vía por donde parece 
reforzarse la explicación sociologista de la dinámica de las teorías científicas; si suponemos que no se dispone de argumentos concluyentes, la tarea de la ciencia se presenta como una actividad social guiada por valores extracientíficos. Pues bien, opino que es precisamente el reconocimiento de la existencia de valores cognitivos concretos y compartidos lo que asegura la existencia de auténticos conflictos morales y políticos cuando se trata de hacer ciencia. Por tanto no parece adecuado invalidar la inducción como mecanismo psicológico irracional o como el lugar por donde penetran toda suerte de valores espurios, sino que es precisamente la existencia de valores cognitivos concretos lo que explica, precisamente porque se atiende a esos valores cognitivos, que la argumentación inductiva aparezca como algo diferente del papel que juegan los intereses políticos, ideológicos, o los intereses de prestigio profesional del investigador, quienes con su presencia dan lugar al conflicto cognitivo. (Cfr. pág. 45 y sgs. de I. Levi Hard Choices.)

Aunque son muchos los pragmatistas que reconocen diversas formas de pluralismo evaluativo, tienden a considerar ese pluralismo sólo en términos morales, éticos, económicos, políticos 0 , en general, prácticos, pero resulta importante reconocer la presencia misma de valores epistémicos. Y, como ha dicho Isaac Levi, en la medida en que hay diversidad de valores hay oportunidad para el conflicto entre valores $y$, aspecto más atinente, hay ocasión para que la decisión se produzca en el ámbito de situaciones de conflicto irresuelto.

Someter nuestras propuestas al test crítico de la falsación es un compromiso cognitivo más, en este caso resultado de una reflexión metodológica. En forma parecida a como Amartya Sen, para el campo de la moral y a partir de sus consideraciones como teórico de la elección racional, ha propuesto una consideración compleja del individuo entendido como un compromiso social pero a la vez con un conjunto de aspiraciones no reductibles a una única medida, a una exclusiva dimensión de utilidad, posibilidad que le ha conducido a estudiar el vector de capacidades potenciales, así 
ocurre con el tema de la ciencia en general para la que no hay que proponer un análisis metodológico unidimensional (por cierto, crítica que molestaba sobremanera a Popper cuando se la planteaba Bar-Hillel).

La posición que adopto respecto a la argumentación es cercana a la de quienes defienden que no se puede hablar seriamente de una argumentación no retórica. La argumentación y la retórica no son dos ámbitos separados, antes al contrario no se puede entender un argumento concreto sin aquellos componentes contextuales que tradicionalmente eran los que se consideraban en el marco de la retórica, además la referencia al problema del tercero que juzga sobre nuestros argumentos o debates (en caso límite el propio sujeto) no es asunto prescindible sino un lugar privilegiado para la aceptación "razonada" del conjunto de conocimientos que queremos explicitar; hay un papel persistente y constante del oyente, de lo subjetivo, sistemáticamente incorporado en el proceso de razonamiento.

Indirectamente ya hemos dicho que el problema de la inducción debe ligarse al problema de la incertidumbre, a la cuestión de cómo razonar en situaciones de incertidumbre. Esta ubicación es tanto como trabajar desde el supuesto de la incompletud de nuestras bases de datos; pero, además, lo inductivo nos aparece ahora relacionado con lo retórico-argumentativo puesto que necesariamente ha de tener en cuenta el proceso concreto.

Tratamos de argumentar y razonar sobre dominios concretos y esto exige de nosotros que hagamos constantes simplificaciones, nos encontramos persistentemente con la necesidad de dejar sin conocer muchos hechos, no decirlos o resumirlos, condensarlos. Como ha dicho J. Pearl: "el arte de razonar bajo incertidumbre equivale al de representar y procesar resúmenes de excepciones". Es interesante tener presente que los sistemas expertos de primera generación en inteligencia artificial habían trabajado con las excepciones, asignando a las proposiciones medidas numéricas que se combinaban de acuerdo con principios de uniformidad sintáctica de manera parecida a cómo los valores de verdad se combina- 
ban en lógica. Ocurre, sin embargo, que las medidas de la incertidumbre se proponen para algo muy diferente de para lo que se proponen los valores de verdad. Debemos tener en cuenta, como dice Pearl, que si los valores de verdad en lógica caracterizan a las fórmulas que discutimos, las medidas de incertidumbre pretenden caracterizar las excepciones, es decir, los hechos que no se muestran en las fórmulas. "Mientras la sintaxis de las formulas es una guía perfecta para la combinación de los visibles, es casi inútil para combinar los invisibles (...) Tales excepciones invisibles pueden interactuar de maneras intrincadas y ocultas, como resultado de todo ello perdemos la mayor parte de los rasgos computacionales atractivos de la lógica clásica: su modularidad y su carácter monotónico".

Se trata de reconocer algo cognitivamente inevitable, aceptar cierto estado de nuestro conocimiento $y$, a partir de ahí, siguiendo mecanismos argumentativos, proponer determinadas conclusiones que incorporamos a nuestro conocimiento compartido. Al razonar siempre estamos aceptando determinado campo de conocimiento como cierto, por tanto no se trata de dar cuenta formal del procedimiento de inducción buscando los elementos de una lógica inductiva que pretenda cubrir o captar esta práctica a partir de los modelos de la deducción, generalización presente en las pretensiones de transformar o reducir la lógica inductiva a la teoría de la probabilidad o de la inferencia estadística. Se trata de pensar en situaciones en las que no se puede prescindir, al menos como punto de referencia último, del individuo como soporte de esos procesos cognitivos.

Una mejor sistematización tiene mas que ver con una forma particular de observar la argumentación y, por supuesto, con lo que se llamaba la lógica del descubrimiento; una forma en la que el papel de lo que llamo el juego retórico está presente porque se trata de encontrar situaciones de equilibrio cognoscitivo en las que encontremos cierto acuerdo y estabilidad en la resolución. Desde esta perspectiva tienen relevancia las teorías de la racionalidad que superen las visiones maximizadoras y optimizadoras; debemos 
atender a otros mecanismos de racionalidad, como son los de racionalidad limitada o racionalidad por satisfacción como en su día propuso $\mathrm{H}$. Simon. Mediante el mecanismo de satisfacción de ciertas constricciones no se busca la optimización de los procesos sino analizar su dinámica particular. Desde luego esto exige trascender la noción formalista de la argumentación e incorporar el tratamiento pragmático de ella, sin perder el objetivo de la "máquina de hacer argumentos". Un elemento central en nuestra noción de argumentación es el elemento intencional, argumenta un individuo del cual presuponemos su racionalidad. Ese elemento intencional es también compartido por el núcleo de la noción de retórica pues, en definitiva, ella es una forma de argumentación que al no ser concluyente parece que depende del poder de persuasión de quien lo sostiene (en la noción de ciencia de Kuhn se incorpora ese elemento persuasivo).

Lo intencional no está necesariamente marcado por lo arbitrario, ni por la arbitrariedad; el elemento nuclear de la noción de inducción puede estar en ese momento de la no arbitrariedad, y aquí es donde aparece el esfuerzo por la objetivación, por pensar que conocemos el campo máximo de lo posible para satisfacer el argumento inductivo (lugar donde se apoya el ataque popperiano).

Una distinción muy importante en el mapa de las situaciones de elección racional es la que se da entre decisiones paramétricas y decisiones estratégicas. En una decisión paramétrica el individuo se enfrenta a un conjunto de restricciones o ligaduras externas que en cierto sentido están "dadas", son "parámetros" de la situación. Por tanto las estima tan bien como puede y decide actuar. ( $¿$ Caso del juez?) Una situación estratégica se caracteriza, no obstante, por la interdependencia de las decisiones (interacción intencional entre agentes intencionales). Antes de poner en práctica su intención, cada agente tiene que anticipar lo que otros probablemente harán, lo que puede requerir por su parte cierta estimación sobre lo que los otros preveran sobre lo que él hará. Sus decisiones entran como parte determinante de las constricciones que conforman su propia decisión. Durante mucho tiempo se ha pensado que 
esto conducía a una serie ilimitada de antecedentes, a una especie de regreso infinito, hasta que se ha demostrado que puede romperse ese regreso mediante la noción de punto de equilibrio. Ese punto viene a ser un determinado conjunto de decisiones (una decisión o estrategia para cada persona que está en la interacción) que tiene la propiedad de que nadie puede mejorar su situacion desviándose de su elección de equilibrio en la medida en que los otros mantengan su elección. Un punto de equilibrio no es sino una situacion (un conjunto de elecciones) que son optimas en su interrelación.

Precisamente la discusión de cuales han de ser las decisiones estratégicas (esas decisiones que están influidas por las decisiones de los otros) es el tema del que se ocupa la teoría de juegos.

Como indica Levi, no siempre debemos pensar que el individuo toma una decisión de acuerdo con el conocimiento completo y por tanto con una total transparencia; es cuestión de analizar situaciones en las que se produce una decisión sin haber resuelto todos los conflictos entre valores éticos, estéticos, cognitivos; buena parte de los problemas que aparecen en los teoremas de imposibilidad de Arrow -estudiados en la teoría de la elección social- pueden ser vistos de otra manera como problemas que surgen en la toma de decisión en situaciones de conflicto. Un caso claro de elección con conflicto irresuelto es el de las situaciones de incertidumbre y un caso elemental de incertidumbre se da en aquellos casos donde con frecuencia decimos que aplicamos el razonamiento inductivo.

Si no podemos garantizar una única explicación científicamente correcta del mundo, entonces tampoco podemos sostener una noción unívocamente correcta de la racionalidad. Podemos hablar de accion intencional, pero lo de racional es otra cuestión. Precisamente al precisar nuestra noción de intencionalidad podría aparecer como complemento retórico el añadido de racional, sin duda un añadido de autoridad.

Así pues parece obvio que hay diversas formas de entender la racionalidad y que esa variedad resulta de enorme importancia para lo que se hace en ciencia social. Todo ello es aún poco 
significativo para lo que supone el auténtico giro retórico. El giro retórico es algo que vaya más allá de una posición sociologista en teoría de la ciencia.

Un ejemplo interesante de los resultados que ha producido en el ámbito de la economía la consciente reflexión retórica, claramente vinculada a Kuhn, es como ya he dicho el trabajo de McCloskey. La discusión interna entre economistas ha sido intensa. Algunos autores, por ejemplo, D. Hausman, han rechazado de plano los argumentos de McCloskey pero valdría la pena mostrar una de sus contribuciones más sugerentes: el "metateorema de la reformulación de los supuestos".

En su libro Knowledge and persuasion in economics, CUP, 1994, dedica Donald McCloskey, un capítulo a lo que llama la retórica del formalismo matemático en economía. Su crítica no es al uso de las matemáticas en economía, más bien se trata de una crítica al tipo de matemáticas que se utilizan. Resulta muy interesante observar cómo, a diferencia del tipo de matemáticas que se emplean en los departamentos de física o de ingeniería, en los cuales el tema de las magnitudes aparece como muy importante y el grado de idealización del modelo aparece como algo determinante, resulta que en los departamentos de ciencia económica el desarrollo principal se dirige hacia un tipo de teorización matemática en la que prevalecen los teoremas de existencia y de unicidad de las soluciones, como si estuvieramos en el mejor de los mundos de la formalización matemática.

Los economistas académicos se han ido olvidando de hablar de las dimensiones de las cosas, han adoptado los valores de ese admirable departamento científico en el cual es irrelevante el tamaño de las cosas y lo que importa es su existencia.

Siguiendo esta línea argumental, McCloskey ha propuesto otro añadido retórico, ahora añadido suyo. Se trata de lo que llama el "Metateorema del hiperespacio de los supuestos", con él pretendo concluir este breve acercamiento a la retórica en la ciencia social: 
"Dado cualquier conjunto de supuestos A que impliquen una conclusión $\mathrm{C}$, para cada conclusión alternativa C'arbitrariamente alejada de $C$ (por ejemplo disjunta de $C$ ), existe un conjunto de supuestos $A^{\prime}$ (arbitrariamente cercanos al supuesto original $A$ ) de manera tal que $A^{\prime}$ implica $C^{\prime \prime \prime}$

El problema es un problema retórico. El prestigio del argumento que se apoya en la matemática ha conducido a los economistas a creer, en contra de su disciplina, que podrían conseguir algo intelectualmente valioso, probando o rechazando en la pizarra grandes verdades sociales. Los programas de investigación vigentes desde los años 40 , que se centraban en los teoremas de existencia han tenido durante largo tiempo éxito retórico, hasta que los economistas se han ido dando cuenta de que después de todo nada se había concluido.

Los economistas se han embarcado en una expedición de pesca en el hiperespacio de los mundos posibles. El problema está en que no han pescado nada con su línea teórica. A pesar de toda la retórica sobre el positivismo, no demuestran la manera de hacer trabajo empírico.

No es que se rechacen los valores que se desarrollan en los departamentos de matemáticas, el problema aparece cuando los científicos sociales abandonan una cuestión social (por ejemplo, una cuestión económica) para dedicarse a una cuestión matemática y se olvidan de volver al departamento de estudios sociales. En nuestro caso eso es tanto como decir que la vieja conexión sobre los sentimientos morales y la economía, en la buena tradición de A. Smith, o los problemas que planteaba Mandeville en la fabula de las abejas siguen presentes y hay que darles solución sabiendo que siempre se trata de una solución que pretende convencer para hacerse eficaz en la práctica social. No dejarse llevar por el imperialismo de cierta teorización económica a la hora de hacer teoría social, sería una buena recomendación, retórica, para quienes se preocupan de teoría política y piensan que la última palabra la tiene el último teorema sobre teoría de juegos. Concluyo pues, sin seguir la recomendación retórica de recapitular los resultados. 
Pienso que algo habremos pescado en este mundo del hiperespacio de los mundos posibles que nos ofrece la lectura retórica de la investigación en ciencias sociales. Existe una imagen de libro de texto, o de mito de la ciencia, que puede ser causa sistemática de confusión (T. S. Kuhn: "La función de la medición en la física moderna" en La tensión esencial). Incluso la presencia de Kuhn en los capítulos metodológicos puede ser una fuente añadida de confusión. No siempre esa presencia es muestra de calidad argumental de la investigación, en muchos casos se ha utilizado como fuente de autoridad, como legitimación previa.

Algo de esto han conseguido descubrir quienes han visto el enorme componente retórico de las ciencias sociales. Estos críticos retóricos hunden sus raíces en la obra de Kuhn y con ello muestran, de paso, que el uso de Kuhn es también plural. 